\title{
Les enquêtes en population générale sur le handicap : un outil d'observation essentiel de la statistique publique
}

Jean-François Ravaud - socio-épidémiologiste

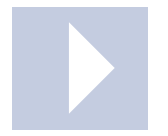

À partir des années 1990, alors que le vieillissement de la population commençait à poser la question de la dépendance et du handicap des personnes âgées, le besoin de données statistiques en la matière est devenu criant. La réalisation d'enquêtes de grande ampleur, représentatives de l'ensemble de la population, a été capitale pour la connaissance des populations handicapées et de leurs conditions de vie. Pourtant, et malgré les recommandations internationales appelant à développer ces enquêtes populationnelles, leur pérennité ne semble pas assurée.

Les problèmes de définition ont longtemps été un obstacle au développement des statistiques sur le handicap. Au début des années 2000, l'Organisation mondiale de la santé (OMS) a apporté des clarifications essentielles avec la Classification internationale du fonctionnement du handicap et de la santé (OMS, 2001), qui sert désormais de référence internationale permettant un langage commun. De même, en France, l'absence de définition légale du handicap a été comblée par la définition fournie par la loi du 11 février 2005 (article 2).

Notre article prend pour référence la conception universaliste du handicap de l'OMS (Bickenbach et al., 1999), selon laquelle le handicap ne désigne pas une population particulière mais un processus qui peut concerner tout un chacun à une période ou une autre de sa vie. Par conséquent, il ne différencie pas dépendance et handicap, mais aborde celui-ci comme un concept générique englobant les notions de dépendance et de perte d'autonomie, lesquelles en sont des déclinaisons particulières pour les personnes âgées.

Après avoir rappelé comment s'est imposée en France la nécessité de compléter les données administratives sur le handicap et de suivre en cela la dynamique internationale, nous montrerons le rôle irremplaçable des 
enquêtes en population générale dans le pilotage de l'action publique. Ces enquêtes se caractérisent par leur ampleur d'un point de vue technique et leur ambition scientifique. Représentatives de l'ensemble de la population française, elles sont souvent adossées au Recensement général de la population et mobilisent des échantillons de grande taille (plusieurs milliers d'individus), permettant d'analyser avec précision les caractéristiques des différentes sous-populations étudiées. La connaissance des personnes handicapées est une bonne illustration de l'intérêt de ces démarches statistiques, comme le montrent les enquêtes dites HID pour « Handicaps Incapacités - Dépendance », réalisées entre 1998 et 2001, et les enquêtes Handicap-Santé réalisées entre 2007 et 2009. Nous terminerons en évoquant l'avenir du système de statistique sur le handicap et ses enjeux.

\section{Compléter les données administratives sur le handicap}

Depuis la loi d'orientation en faveur des personnes handicapées de 1975, le handicap est un volet important du système français de protection sociale. Chaque domaine couvert (scolarisation, emploi, prestations, établissements spécialisés...) fait l'objet d'un recueil de données statistiques d'origine administrative sur le handicap qui ont essentiellement pour objectif de décrire l'activité des dispositifs mis en place, de caractériser le système de prise en charge et de renseigner les dépenses publiques qu'il entraîne.

Toutefois, dans les années 1980, les difficultés d'estimation de la population handicapée ont commencé par apparaître comme un des obstacles majeurs à l'élaboration des politiques sociales. Combien y a-t-il de personnes handicapées en France ? Posée de façon récurrente dans les rapports officiels sur le handicap, cette question exprimait une demande sociale non satisfaite. Face au labyrinthe que constitue le système d'information statistique français, les efforts déployés pour recenser les approches existantes et les articuler échouaient à en faire l'assemblage. Les rares tentatives de synthèse montraient que les données statistiques existantes étaient, selon les questions traitées, insuffisantes, pléthoriques ou inadaptées (Triomphe, 1995).

Les différentes ressources statistiques publiques sur le handicap se distinguent en fonction du type d'aide ou de prise en charge dont bénéficient les individus. Les personnes considérées handicapées (ou dépendantes), parce qu'elles bénéficient d'une prise en charge par un établissement spécialisé, sont étudiées dans plusieurs enquêtes menées par la Direction de la recherche, de l'évaluation, des études et des statistiques (Drees) du ministère des Affaires sociales et de la Santé. Les enquêtes auprès des établissements et services pour enfants et adultes handicapés, dites enquêtes «ES handicap » (pour « établissements sociaux »), renseignent depuis trente ans sur les utilisateurs d'établissements médico-sociaux. La Statistique annuelle des établissements de santé (SAE) renseigne depuis 1995 sur les établissements psychiatriques publics et privés. Enfin, l'enquête auprès des établissements d'hébergement 
pour personnes âgées (EHPA) couvre depuis 1985 les établissements pour personnes âgées. Les personnes bénéficiant de prestations en espèces ou d'avantages pécuniaires, pour leur part, sont dénombrées par chaque régime de financement. Ainsi, les enquêtes sur la Prestation de compensation du handicap (PCH), l'Allocation compensatrice tierce personne (ACTP) et l'Allocation personnalisée d'autonomie (Apa) sont menées notamment auprès des conseils généraux. Mais les régimes sont multiples : Maisons départementales des personnes handicapées (MDPH), régimes de Sécurité sociale, invalidité de guerre, aide sociale, assurances, avantages fiscaux... En outre, compte tenu de son parcours de vie, il n'est pas rare qu'un individu cumule plusieurs prestations, ce qui complique encore l'appréhension du système d'aide.

Enfin, la scolarisation et l'emploi des personnes handicapées sont l'objet de recueils de données spécifiques. La Direction de l'évaluation, de la prospective et de la performance (Depp) du Ministère de l'éducation nationale mène des enquêtes sur la scolarisation des enfants handicapés. La Direction de l'animation de la recherche, des études et des statistiques (Dares) du ministère du Travail et de l'Emploi analyse les données sur l'emploi des travailleurs handicapés issues de la Déclaration annuelle obligatoire d'emploi des travailleurs handicapés (DOETH), dont la gestion a été transférée en 2013 à l'Association de gestion du fonds pour l'insertion professionnelle des personnes handicapées (Agefiph).

Chacune de ces enquêtes contribue de façon significative à l'estimation, peu aisée, de la population handicapée. Toutefois, ce tour d'horizon montre combien ces statistiques, morcelées et fragmentaires, ne permettent pas de connaître, notamment, les caractéristiques des personnes qui ne bénéficient pas des aides publiques que leur situation justifierait. Une politique sociale qu'on voudrait adaptable aux besoins ne peut pas se fonder sur des données cantonnées à la seule description du système de prise en charge existant, qui ne permet pas d'avoir une vision prospective des besoins sociaux.

Plusieurs questions très importantes sont ainsi absentes des données recueillies dans les dispositifs décrits ci-dessus, à commencer par la description des incapacités et la situation des personnes handicapées, c'est-àdire leur environnement humain, physique, technique et économique. La deuxième est l'évaluation du non-recours. Celle-ci est impossible en l'absence d'une mesure des besoins et des caractéristiques de la population vivant avec un handicap. Par exemple, on ne peut pas déterminer si les bénéficiaires de prestations ou d'une prise en charge institutionnelle présentent des caractéristiques différentes de celles des personnes handicapées qui n'en reçoivent pas. Le troisième sujet non traité est celui des modalités d'adaptation des établissements et des prestations à l'état de santé et à la situation réelle de l'ensemble des personnes handicapées.

Il y a vingt ans, l'information statistique française sur le handicap souffrait 
d'un déficit de données nationales couvrant toute la population, d'un déficit de données représentatives et d'absence de possibilités de prévisions. Elle fut alors qualifiée de "puzzle incertain» (Mormiche, 1998). C'est dans ce contexte qu'a émergé la demande sociale de disposer, pour les nombreuses données statistiques d'origine administrative, de données de cadrage qui soient indépendantes du système d'aide.

\section{Les enquêtes populationnelles}

Les réflexions menées au cours des années 1990 ont abouti à un double constat : celui du retard de la France, notamment par rapport aux pays anglosaxons, dans le domaine des statistiques nationales sur le handicap, l'incapacité et la dépendance et celui de l'hétérogénéité et de l'insuffisance de l'appareil d'information alors concerné. Si la prise en compte des situations de handicap a commencé à figurer dans certaines enquêtes de l'Insee, par exemple l'enquête Conditions de vie (1), la réalisation d'une enquête de grande ampleur apparut comme une nécessité pour mieux connaitre les situations de handicap. Couvrant l'ensemble de la population française, à tous les âges et dans tous les lieux de vie (domicile ou institution), cette enquête en population générale devait se centrer sur les incapacités et ne pas séparer les domaines du handicap et de la dépendance. Ses objectifs étaient de mesurer la prévalence des handicaps, d'évaluer les flux d'entrée et de sortie en incapacités et d'étudier les aides existantes et les besoins non satisfaits.

\section{Les enquêtes pionnières : Handicaps - Incapacités - Dépendance (HID) 1998-2001}

Le lancement des enquêtes HID fut précédé d'une phase de concertation. Des études de faisabilité furent effectuées en 1994-1995 par la Mission interministérielle pour la recherche (Mire) du ministère des Affaires sociales, associée à un comité d'experts. Une consultation des différents acteurs sociaux et du milieu de la recherche concernés fut également entreprise (Letourmy, 1998). À la suite des recommandations du Conseil national de l'information statistique (Cnis, 1997), l'Insee entreprit une opération d'envergure en se lançant dans un nouvel ensemble d'enquêtes avec le soutien financier des principaux acteurs concernés ${ }^{(2)}$. Cette opération permit une collaboration inédite entre l'Insee et l'Inserm dans le domaine des statistiques de santé. D’une manière générale, les enquêtes HID été rendues possibles grâce à un large partenariat institutionnel et scientifique (3), coordonné par Pierre Mormiche de l'Insee.

Le recueil des données des enquêtes HID (Handicaps - Incapacités Dépendance), réalisé par l'Insee, s'est déroulé de 1998 à 2001. Ces enquêtes nationales représentatives ont couvert la population vivant à son domicile (enquête HID-Ménages 1999) et celle vivant en institutions pour personnes âgées et pour personnes handicapées (enfants et adultes), dans des services 
hospitaliers de long séjour et des services ou établissements psychiatriques (enquête HID-Institutions 1998). Les informations collectées ont concerné aussi bien la description des déficiences que les incapacités et leurs origines, l'environnement sociofamilial et architectural ainsi que les différentes sphères de la participation sociale des personnes.

Ces enquêtes, pionnières en France, s'appuient sur plusieurs choix essentiels. Suivant les recommandations de l'Onu édictées à cette époque (OnuStat, 1996) et s'appuyant sur l'expérience internationale, le passage de l'enquête HID dans les ménages ordinaires a été précédé d'une phase dite de filtrage mais non sélective, au sens où elle n'a pas exclu du champ de l'enquête les personnes qui ne correspondaient pas à une définition du handicap préétablie par les concepteurs de l'enquête. Ce choix a ainsi permis d'aborder le handicap comme une réalité multiforme en en multipliant les angles d'approche (Ravaud et al. 2002) tout en conservant une population témoin dans l'échantillon final. Par ailleurs, grâce à un second passage effectué deux ans après pour étudier l'évolution des situations, ces enquêtes ont permis de déterminer les flux de personnes nouvellement handicapées ou à l'inverse celles qui ne le sont plus (du fait d'une rémission par exemple). Elles ont permis d'estimer que si, $40 \%$ de la population française présente une déficience, $21,1 \%$ ont au moins une incapacité, 11,7 \% sont limités dans leurs

$\boldsymbol{6}$ Couvrir, avec un questionnement homogène, toute la population à tous les âges et, surtout, dans tous les lieux de vie permet de redonner tout son sens à la notion de «population générale » (...). activités, 9,5\% se considèrent handicapés, 6,2\% mentionnent un type d'aide quelconque et 5,2\% sont reconnus socialement comme handicapés par le biais d'une reconnaissance administrative (Ravaud et al. 2002).

Couvrir, avec un questionnement homogène, toute - 9 la population à tous les âges et, surtout, dans tous les lieux de vie permet de redonner tout son sens à la notion de «population générale », les institutions ayant été jusqu'alors exclues du champ des enquêtes de l'Insee. Privilégier les déclarations de la personne et ne s'adresser à un proxy qu'en seconde intention nécessite en outre de dépasser les limites des méthodes de collecte traditionnelles dans les cas où les personnes ont des besoins spécifiques (de communication, par exemple, dont la non-prise en compte les fait aisément considérer comme « inaptes à répondre »). Ces innovations sont des sources de réflexion utiles sur la méthodologie des autres enquêtes de l'Insee et leur " accessibilité » aux personnes handicapées.

Last but not least, les enquêtes HID ont eu un rôle rassembleur dans le champ de la recherche en santé publique et en sciences sociales sur le handicap, ce qui a eu un effet très structurant pour la communauté scientifique et les acteurs sociaux. L'animation scientifique autour des exploitations des données a été effectuée dans le cadre de plusieurs séminaires et colloques (4). Une première synthèse des résultats de l'enquête a fait l'objet d'un numéro double de la Revue française des affaires sociales (RFAS, 2003). 


\section{Les Enquêtes Handicap-Santé 2008-2009 : vers une stabilisation du dispositif?}

Assez logiquement, le succès de la première opération, menée avec les enquêtes HID, a posé la question de son renouvellement. Deux lois importantes marquent les années 2000 pour la France : la loi de santé publique du 9 août 2004, qui fixe dans son annexe des objectifs précis à atteindre à l'horizon 2008 en matière de statistiques de santé, notamment une liste d'indicateurs à mesurer tous les ans ou tous les cinq ans, et la loi du 11 février 2005 sur l'égalité des droits et des chances, la participation et la citoyenneté des personnes handicapées.

Au plan international, l'OMS révise la première classification internationale du handicap (cadre théorique de référence lors des enquêtes HID) qui devient en 2001 la Classification internationale du fonctionnement du handicap et de la santé (OMS, 2001). Par ailleurs, Eurostat engage une réflexion sur l'harmonisation des dispositifs statistiques européens et recommande l'introduction d'un questionnement commun, European Core Health Interview Survey (Echis), dans les enquêtes santé des États membres de l'Union européenne.

Tenant compte de cette évolution du contexte, l'enquête Handicap-Santé est lancée en 2005. L'orientation suivie par la Drees et l'Insee qui en assurent la maitrise d'ouvrage est alors celle d'une fusion de l'Enquête décennale sur la santé et les soins médicaux initiée en 1960, dont la dernière édition date de 2003, et des nouvelles Enquêtes HID en une enquête quinquennale comportant un tronc commun avec, tous les cinq ans, une dominante qui alternerait santé et handicap (Bouvier, 2011).

Les objectifs des enquêtes Handicap-Santé sont de mesurer le nombre des personnes en situation de handicap, d'évaluer le « désavantage » vécu par elles, de relever la nature, la quantité et l'origine des aides existantes ainsi que les besoins non satisfaits, et de mieux connaitre les aidants. Pour évaluer l'évolution du handicap et de la dépendance, il faut que les enquêtes soient dans une large mesure comparables avec les enquêtes HID. Le volet santé doit ainsi permettre de suivre certains indicateurs majeurs de santé publique et d'intégrer les différents modules européens de l'enquête l'Echis. En revanche, l'objectif d'évaluer les flux d'entrée et de sortie en incapacité avec deux vagues d'enquêtes est abandonné.

Deux projets d'appariement sont approuvés par la Commission nationale de l'informatique et des libertés (Cnil). Le premier concerne les données de l'état civil et le Répertoire national d'identification des personnes physiques (RNIPP) afin d'évaluer les inégalités de mortalité et d'espérance de vie selon la présence éventuelle, la nature et la sévérité d'un handicap, et d'étudier les causes de décès. Le second projet porte sur le fichier SNIIR-AM (Système national d'information interrégimes de l'assurance maladie) de la Cnamts afin de connaitre les consommations de soins des répondants à l'enquête. 
Comme les enquêtes HID, les enquêtes Handicap-Santé ont mis en jeu plusieurs dispositifs de recueil d'information :

- l'enquête Vie quotidienne et Santé (VQS 2007) a été effectuée au moyen d'un questionnaire court par voie postale auprès d'un échantillon de 263000 personnes ;

- l'enquête Handicap-Santé Ménages (HSM 2008) a porté sur un échantillon de 39000 personnes vivant à leur domicile, tirées au sort à partir de la base de sondage constituée grâce à l'enquête VQS en surreprésentant les personnes présumées handicapées ;

- l'enquête Handicap-Santé Aidants (HSA 2008) a été menée en 2008 auprès de 5000 aidants informels repérés dans le cadre de l'enquête HSM. Elle permet de mieux connaitre les différentes formes d'aide, les motivations et manifestations du soutien informel, l'articulation de celui-ci avec l'aide professionnelle et la qualité de vie des aidants.

- l'enquête Handicap-Santé Institutions (HSI 2009) auprès de 9000 personnes tirées au sort dans 1500 structures sanitaires, médico-sociales et sociales (établissements d'hébergement pour personnes âgées, établissements d'hébergement pour adultes handicapés, services de psychiatrie, centres d'hébergement et de réinsertion sociale).

Le questionnaire des enquêtes aborde la santé (état de santé général, recours aux soins, nutrition, maladies), le handicap (déficiences, limitations fonctionnelles, restrictions d'activité, aides techniques, aides humaines de l'entourage ou de professionnels), la participation à la vie sociale, les discriminations, l'accessibilité du logement, les revenus et allocations, la scolarisation, l'emploi, l'environnement familial. Il permet de renseigner plusieurs échelles d'évaluation comme celle de Katz ou les niveaux de dépendance de la grille Aggir.

S’appuyant sur l'expérience acquise par sa contribution antérieure à la conception puis à l'animation des exploitations et aux analyses des enquêtes HID, l'Institut fédératif de recherche sur le handicap (IFRH) a été chargé de la coordination des exploitations des enquêtes Handicap-Santé. Depuis 2011, il organise un séminaire mensuel qui réunit les différentes équipes utilisatrices de ces enquêtes ; en juin 2013, il a organisé avec la Drees un colloque, « Handicap et autonomie : la diversité des apports des enquêtes HandicapSanté » dont les actes sont en cours de publication (5).

\section{Quel avenir pour le système} statistique public sur le handicap?

Ces enquêtes populationnelles ont été essentielles pour la connaissance de la (des) population(s) handicapée(s) et de ses (leurs) conditions de vie. Une dynamique d'exploitation remarquable s'est mise en place autour d'elles. Mais, après une enquête pionnière puis une enquête de stabilisation, le dispo- 
sitif se trouve actuellement à un tournant. Assimilable à une «très grande infrastructure » pour les chercheurs, ce type d'opération nécessite un accord très large des acteurs sociaux, des organismes susceptibles de le porter et des équipes susceptibles d'en faire l'analyse (ONFRIH, 2011). D'ores et déjà, le projet d'enquête quinquennale prévu en 2008 est devenu caduc. La nouvelle enquête Care prévue pour 2015 a une philosophie très différente : centrée sur les seniors et l'évolution de la dépendance et du reste à charge des personnes, elle ne couvrira pas l'intégralité de la population, mais uniquement les plus de 60 ans. Alors que son avenir est actuellement incertain, la pérennité d'une grande enquête nationale sur le handicap et la perte d'autonomie auprès de la population générale au-delà des barrières d'âge reste sans conteste un enjeu majeur pour la statistique publique qui ne peut se contenter des statistiques administratives des dispositifs impliqués dans le champ.

Les deux vagues d'enquête présentées ci-dessus laissent de nombreuses questions en suspens. La moindre n'est pas l'échelle géographique, qui doit s'étirer entre les exigences de comparaisons internationales et les demandes croissantes de représentativité des données au niveau local, en particulier pour fournir une information pertinente aux conseils généraux en charge de l'aide sociale. Une autre tension stratégique se fait sentir entre le besoin de grandes enquêtes de cadrage dédiées à la thématique du handicap et la nécessité d'introduire un module handicap dans des enquêtes sectorielles ordinaires (transports, patrimoine, emploi, etc.). Enfin, outre des données individuelles, l'approche contemporaine du handicap nécessite des données environnementales pour pouvoir suivre les avancées des politiques d'accessibilité.

Il convient de rappeler, pour conclure, que si le développement d'enquêtes sur le handicap répond à de nouveaux enjeux de santé publique et à l'approche de la santé et du fonctionnement humain promue actuellement par l'OMS, il répond également aux attentes désormais exprimées par la Convention relative aux droits des personnes handicapées adoptée par l'Onu en 2006 et ratifiée par la France en 2010. Dans son article 31, intitulé Statistiques et collecte des données, la Convention stipule que les États parties s'engagent à recueillir des informations appropriées, y compris les données statistiques et résultats de recherche, qui leur permettent de formuler et d'appliquer des politiques visant à donner effet à la Convention. La Convention de l'Onu met donc clairement l'accent sur la nécessité de recueillir des données pour évaluer l'évolution de la situation des personnes handicapées et pour identifier les obstacles qu'elles rencontrent dans l'exercice de leurs droits. 
1 - Après avoir réalisé en 1978-1979 une première étude sur les conditions de vie, qui montrait la nécessité d'une approche globale de la question des inégalités, I'Insee a réalisé en 1986-1987 une enquête sur les Conditions de vie (ECV), baptisée "Enquête situations défavorisées », qui mettait l'accent sur le cumul des inégalités.

2 - Financement par le ministère de l'Emploi et de la Solidarité, les caisses nationales de sécurité sociale (Cnamts, Cnav, Cnaf et Canam), des caisses de retraite complémentaire (Agirc, Arrco), des mutuelles et assurances (Fédération française des sociétés d'assurance, Mutuelle générale de l'éducation nationale...), I'Association de gestion des fonds pour l'insertion professionnelle des personnes handicapées (Agefiph) et l'Association des paralysés de France (APF).

3 - Institut national des études démographiques (Ined), Institut national de la santé et de la recherche médicale (Inserm), Instituts fédératifs de recherche sur le handicap et Vieillissement, Santé, Société, la Drees, le Centre de recherche, d'étude et de documentation en économie de la santé (Credes), le Centre technique national d'études et de recherche sur les handicaps et inadaptations (CTNERHI).

4 - Une abondante documentation sur les méthodes, les questionnaires et les publications réalisées est accessible sur le site de l'Institut fédératif de recherche sur le handicap : http://ifr-handicap.inserm.fr

5 - Les questionnaires des différentes enquêtes Handicap-Santé sont disponibles sur le site de la Drees (http://www.drees.sante.gouv.fr/les-enquetes-handicap-sante,4267.htm/) et les différentes publications effectuées sur le site de l'IFRH (http://ifr-handicap.inserm.fr/).

\section{Bibliographie}

- Bickenbach J.-E., Chatterji S., Badley E.-M. et Üstün T. B., 1999, "Models of disablement, universalism and the international classification of impairments, disabilities and handicaps ", Social Science \& Medicine, vol. 48, n 9, p. 11731187.

- Bouvier G., 2011, «L'enquête Handicap-Santé. Présentation générale », Insee, Document de travail, $\mathrm{n}^{\circ} \mathrm{F} 1109$, octobre.

- Conseil national de l'information statistique (Cnis), 1997, Handicap et dépendance : I'amélioration nécessaire du système statistique, rapport $\mathrm{n}^{\circ} 35$.

- Letourmy A., 1998, "La préparation d'une enquête sur les handicaps », Revue française des affaires sociales, vol. 52, n 1, p. 27-33.

- Mormiche P., 1998, "L'information sur le handicap et la dépendance en France : dépasser le stade du puzzle incertain ", Revue française des affaires sociales, $n^{\circ} 52$, p. 35-42.

- Organisation mondiale de la santé (OMS), 2001, Classification internationale du fonctionnement du handicap et de la santé (Cif), Genève.

- ONFRIH, 2011, Rapport triennal de l'Observatoire national sur la formation, la recherche et l'innovation sur le handicap, rapport à la ministre des Solidarités et de la Cohésion sociale, Synthèse et préconisation, Paris, La Documentation française, Collection des rapports officiels.

- Organisation des Nations unies, 1996, Manual for the development of statistical information for disability programmes and policies, New York, UN Publication, Onu-Stat. 
- Ravaud J.-F., Letourmy A. et Ville I., 2002, « Les méthodes de délimitation de la population handicapée : I'approche de l'enquête de l'Insee. Vie quotidienne et santé », Population, vol. 57, n³ 3 , p. 541-565.

- Revue française des affaires sociales (RFAS), 2003, Handicaps, incapacités, dépendance, janvier - juin, vol. 57, $\mathrm{n}^{\circ} 1-2$.

- Triomphe A. (dir.), 1995, Les personnes handicapées en France. Données sociales 1995, Paris, Inserm-CTNERHI/Puf, 2e édition, nº 212. 